Article

\title{
Ang Pilosopiya ng Laman ni Maurice Merleau-Ponty
}

\section{Christian Joseph C. Jocson and Marvin Einstein S. Mejaro}

\begin{abstract}
Merleau-Ponty's phenomenology opens up the interpretation that human beings are social and intersubjective by nature. First, his concept of the flesh presents a solution against the solipsistic tendency of Cartesian philosophy. The perception of one's own being implies a perception of the shared flesh. Even the perception of the pain of another person elicits a feeling of pain that is not totally alien to one's own flesh. Second, the concept of perception is a dialectic between proximity and distance, such that perception of the being of another person is not solely given through proximity, but perception is primarily characterized by distance. To perceive is to perceive at a distance, never imposing one's structure of understanding or categories to the object of perception, but letting the thing be itself. Furthermore, this perceptual distance can also be interpreted as an ethical distance that allows the Other to be free from the confines of one's own perception.
\end{abstract}

Keywords: Merleau-Ponty, ethical distance, flesh, perception

\section{Introduksyon}

sa sa pinakamahalagang ideya na binahagi sa atin ng penomenolohiya ni Maurice Merleau-Ponty ay ang pagkakaintindi na ang kalayaan ay hindi -nagpapahayag ng pagkasarili kung hindi isa itong pagtuklas ng ating pagiging umiiral-na-nilalang-sa-mundo kasama ang ibang katawang umiiral-na-mga-nilalang-sa-mundo.

Katunog sa konsepto ng kalayaan na ipinakita ni G.W.F. Hegel sa kaniyang Phenomenology of the Spirit: nagiging malaya ang tao na nanahanan sa mundo. Ngunit, upang itala ang nasabi ni Hegel, kapag sinabing 'pagtahan sa mundo,' hindi nito kubling pinapahayag na lumilikha ang isang tao ng mundo na wangis sa kaniya; o bilang kaniyang pagmamay-ari. Ngunit, ang 'pagtahan sa mundo' ay pagiging-kaisa at malayang pagkikipagugnayan sa

(c) 2017 Christian Joseph C. Jocson and Marvin Einstein S. Mejaro

https://www.kritike.org/journal/issue 21/jocson\&mejaro december2017.pdf

ISSN 1908-7330

(cc) $\mathrm{BY}-\mathrm{NC}-\mathrm{ND}$ 
iba pang mga tao at ng pananahan sa mundo kasama ang ibang mga tao. Sa pagsunod sa gayong ideya, ipinapamalas ni Merleau-Ponty na ang kalayaan ay hindi dapat mahigpit na pinipigilan ng presensiya ng ibang mga pag-iral. Hidwa sa posisyong antagonistiko ni Jean Paul Sartre na 'impiyerno ang ibang tao.' Tinatalakay ni Merleau-Ponty ang isang ideya ng kalayaan na kadaloy sa ideya ni Hegel ng pagiging-bukas na malaya at nagpapaanyaya sa presensiya ng ibang-mga-umiiral-na-nilalang.

\section{Laban sa Solipsismo}

We have here a dual being, where the other is for me no longer a mere bit behavior in my transcendental field, nor I in his; we are collaborators for each other in consummate reciprocity. ${ }^{1}$ (Dito natutuklasan natin ang pagiging nilalang sa dalawang aspekto, na kung saan ang ibang tao ay hindi nananatiling sa labas ng aking sarili at sa katulad din na paraan, ang aking sarili ay hindi nananatili sa labas ng ibang tao, sapagkat, ang bawat isa sa amin, ay nakikibahagi sa bawat isa sa isang proyekto ng relasyon ng paglalaman ng bawat isa.)

Isa sa mga panimulang punto ni Merleau-Ponty sa kaniyang pilosopiya interkorporeal na katawan ay ang pilosopiya ni Rene Descartes. Proyekto ni Merleau-Ponty ay maibaling ang pag-iisip na ang tao ay naninirahan lang sa sarili niyang pag-iisip. Dagdag pa niya, ang ibang tao na naninirahan sa mundo, ay hindi lang isang likha ng ating imahinasyon, sapagkat naniniraban sila sa mundo kasama natin. May persepsyon ako hindi lamang ng mga ideya o sariling ilusyon ngunit ang persepsyon mismo ng mga bagay na ito ng daigdig. Batid ko na ang siyang nararanas ay ang buhayna-mundo na puno rin ng mga buhay na pagdanas ng maraming mga buhay na laman na nakapaligid sa akin.

Our own body is in the world as the heart is in the organism: it keeps the visible spectacle constantly alive, it breathes life into it and sustains it inwardly, and with it forms a system. (Ang ating katawan ay nasa mundo katulad ng puso sa isang nilalang. Ito ang nagpapanatili na laging buhay ang mga bagay na maaaring masilayan. ${ }^{2}$

${ }^{1}$ Maurice Merleau-Ponty, Phenomenology of Perception, trans. by Colin Smith (London; New York: Routledge, 1958), 413. Henceforth cited as PP.

2 Ibid., 235.

(c) 2017 Christian Joseph C. Jocson and Marvin Einstein S. Mejaro https://www.kritike.org/journal/issue 21/jocson\&mejaro december2017.pdf

ISSN 1908-7330 


\section{PILOSOPIYA NG LAMAN NI MERLEAU-PONTY}

“Ano ang laman ng iyong laman? Ang laman lang ba ng iyong laman ay iyong sariling laman?" Isa sa mga sinusuri ni Merleau-Ponty sa kaniyang pilosopiya ay ang kinagawiang pagtingin sa konsepto ng sarili na tungkol lamang sa sarili at naiisang-tabi ang anomang tungkol sa ibang mga nilalalang. Katulad din ng ating pananaw tungkol sa iba, hinihiwalay natin ang anomang may kinalaman sa sarili. Ang layon ni Merleau-Ponty sa kaniyang ideya ng mapag-ugnay na paglalaman ay maipakita ang sarili at ang iba ay hindi mga tunay na magkasalungat, sapagkat ang sarili at ang iba ay dapat maging bukas sa bawat isa upang maging tunay na kamalayan ng sarili at iba.

Things are an annex or prolongation of Itself; they are encrusted into its flesh, they are part of its full definition; the world is made up of the same stuff as the body. ${ }^{3}$ (Ang mga bagay ay mga nagpapalago at nagpapalayo ng maaring matanaw ng kaniyang sarili, sila ay nakapaloob sa kaniyang laman. Ang mga bagay na ito ay bahagi ng kabuoang kahulugan niya; isang katangian ng mundo ay binubuo ito ng katulad sa ating mga katawan).

Para kay Merleau-Ponty ang matikas na pagsasabi na ang aking katawan ay sa akin lang ay isang maling pananaw. Ang tunay na laman ay nilalaman ng ibang laman at naglalaman ng ibang laman. Sa ibang salita, ang tinatagurian natin na laman ay hindi pagmamay-ari ng sarili o ng iba. Pinapahayag ng ideya ng laman na ang pagiging ng isang nilalang ay lagpas sa kaniyang tinatanaw na sarili. "A proper conception of self and other must not be grounded on the idea of exclusion but instead it must resound inclusion." Mas mabuti na ang ang sariling kamalayan ay hindi lamang simpleng kamalayan na nasa kontemplasyon ng sarili nitong pusod. ${ }^{4}$

Solitude and communication cannot be the two horns of a dilemma, but two 'moments' of one phenomenon, since in fact other people do exist for me. ${ }^{5}$ (Ang pag-iisa at pakikipagtalastasan ay hindi mga magkasalungat na mga bagay, ngunit ang dalawang ito ay bahagi ng isang

\footnotetext{
${ }^{3}$ Maurice Merleau-Ponty, "Eye and Mind," in The Primacy of Perception ${ }_{\llcorner}$ed. by James M. Edie (Illinois: Northwestern University Press, 1964), 163. Henceforth cited as $\operatorname{Pr} P$.

4 Peter Singer, Hegel: A Very Short Introduction (Oxford: Oxford University Press, 2001), 75.

${ }^{5}$ Merleau-Ponty, $P P, 418$.

(c) 2017 Christian Joseph C. Jocson and Marvin Einstein S. Mejaro https://www.kritike.org/journal/issue 21/jocson\&mejaro december2017.pdf ISSN 1908-7330

(c) BY-NC-ND
} 
pangyayari, dahil ang ibang tao ay tunay na nadarama ko.)

Para kay Merleau-Ponty, kung ang pang-unawa ko sa isang penomenolohikal na laman ay nasa hangganan lamang ng aking sarili, kung sa gayon, hindi ito ang angkop na laman sa isang penomenolohikal na bagay. "Ang laman na nalalaman lang ang sariling laman ay wala talagang nalalaman.” Ang kaalamang paglalaman ay laging nagpapahayag ng paguugnay, laging tumatawag sa iba.

Ang naging dahilan ng trahedya ni Narcissus ay hindi dahil masyado niyang minahal ang kaniyang sarili, ngunit dahil inipit niya ang kaniyang sarili ay sarili lang niyang larawan. Kapag nararanasan ng taong harapin sa salamin ang kaniyang sariling repleksyon, dapat niyang maabot ang pananaw na mayroong mundo na kasama sa kaniyang repleksyon. Mahalaga rin na bigyan ng pansin na ang mundo na kasamang nasisilayan sa sariling repleksyon ay nagpapahiwatig na ang tinatawag na sarili ay bahagi ng mundo at hindi ang mundong bahagi lang ng sariling repleksyon. Ang tunay na nilalang na naglalaman ay nakikita ang larawan na lumalagpas at umaapaw sa sarili niya - waring unang patikim lang ng tunay na kamalayan ng paglalaman. ${ }^{6}$

My flesh is of a piece with that of both things and other persons: "That is why we say that in perception the thing is given to us 'in person,' or "in the flesh.'"7 (Ang aking laman ay bahagi ng mga bagay ng ibang mga tao. Ito ang dahilan kung bakit ang pagdama ng isang bagay ay nararanasan natin sa katauhan niya mismo o sa laman mismo.)

Ang pagiging isang nilalang na nanglalaman ay maaring maabot lang sa pakikipag-ugnayan sa mundo kasama ang ibang mga nilalang na naglalaman. Katulad ng sinabi ni Fichte, nagiging tao lang ang isang tao kapag kasama niya ang iba pang mga tao. ${ }^{8}$ Sinundan ito ni Merleau-Ponty at pinahayag naman niya, na sa pamamagitan ng paglayo sa sarili, mas

${ }^{6}$ Inasmuch as he (Narcissus) had life, he always had the 'whole world' in his flesh. Frank J. Macke, "Seeing Oneself in the Mirror: Critical Reflections on the Visual Experience of the Reflected Self," in Journal of Phenomenological Psychology, 36:1 (2005), 37.

${ }^{7}$ Merleau-Ponty, PP, 320; cf. Merleau-Ponty, $\operatorname{Pr} P, 163$. Quoted in Andrew Cutrofello, Continental Philosophy (New York; London: Routledge, 2005), 67.

${ }^{8}$ J. G. Fichte. Foundations of Natural Right, ed. by F. Neuhouser, trans. by M. Baur (Caabridge: Cambridge University Press, 2000), 37 as cited in Robert Stern, “Is Hegel's MasterSlave Dialectic a Refutation of Solipsism?" in British Journal for the History of Philosophy, 20:2 (2012), 355.

(c) 2017 Christian Joseph C. Jocson and Marvin Einstein S. Mejaro https://www.kritike.org/journal/issue 21/jocson\&mejaro december2017.pdf

ISSN 1908-7330 


\section{PILOSOPIYA NG LAMAN NI MERLEAU-PONTY}

magkakaroon siya ng kamalayan ng kaniyang sarili. Ngunit, ang pagkakataon na makalayo tayo sa ating sarili ay hindi dahil sa sarili nating kakayahan. Sa pamamagitan ng napagpapalayang-presensiya ng ibang tao nagiging posible ang kamalayan ng ating sarili.

As such, consciousness starts from a position where it does not fully understand itself, consciousness is, initially, alienated from itself. The phenomenological development of consciousness discloses the logical journey consciousness must take to overcome its selfalienation. ${ }^{9}$ (Ang kamalayan ay nag-uumpisa mula sa bahagi na hindi niya tunay na naiintindihan ang kaniyang sarili; kamalayan na napalayo sa kaniyang sarili. Ang penomenolohikal na kaunlaran ay isang paglalakbay ng kamalayan mula sa kaniyang pagkaligaw.)

Sa pag-iisip na ang tinatagurian ko na aking laman ay hindi naabutan ko ng buong-buo na aking nalalaman, nagkakaroon ako ng pagkakataon na makita ang aking sarili na hindi lang umiikot sa sarili kong laman. Sa ibang salita, sa pamamagitan ng hindi nagiging ganap ang pagpapakita ng aking sariling laman, nagiging posible ang muling pagkatuklas ang pagkilala sa aking sariling laman. Katulad ng pinapahayag ni Hegel, na ang katanagian ng espiritu na maligaw, malayo, at mawala ang sarili upang muling matagpuan ulit. ${ }^{10}$

Isang magandang halimbawa na maaaring ipakita rito ay ang kapag nanonood tayo ng isang pelikula at makita na nabaril ang protagonista, nasaksak, o nakaranas ng anomang uri ng karahasan. Mapapansin natin na parang nagkakaroon din tayo ng karanasan na nararanasan rin natin ang paghihirap na nangyayari doon sa tauhan sa pelikula. Na masasabi natin na kahit ang sakit na nararanasan ay hindi atin ngunit sa ating sariling laman nararanasan natin na hindi iba ang sakit ng ibang tao sa atin.

Adam Smith already recognized in the 1750s that people naturally respond to the others with gestures appropriate to the person suffering: "When we see a

\footnotetext{
9 Gavin Rae, "Hegel, Alienation, and the Phenomenological Development of Consciousness," in International Journal of Philosophical Studies, 20:1 (2012), 24.

${ }^{10}$ G.W.F. Hegel, Introduction to the Lectures on the History of Philosophy, trans. by T.M. Knox and A.V. Miller (Oxford: Oxford University Press, 2003), 80 as cited in Rae, "Hegel, Alienation, and the Phenomenological Development of Consciousness," 30.

(c) 2017 Christian Joseph C. Jocson and Marvin Einstein S. Mejaro https://www.kritike.org/journal/issue 21/jocson\&mejaro december2017.pdf ISSN 1908-7330

(c) BY-NC-ND
} 
stroke aimed and just ready to fall upon the leg or arm of another person, we naturally shrink and draw back our own leg or our own arm."11 (Nagawang matuklasan ni Adam Smith na noong 1750s na ang mga tao ay may likas na reaksyon sa pagdurusa ng ibang mga tao na matutunghayan sa mga galaw at mga senyales ng katawan. Kunyari naiisip na natin ang isang bagay na babagsak at makasasakit sa binti o braso ng isang tao, napapansin natin na may likas tayo na reaksyong pakiramdaman at ilayo ang sarili nating binti o braso.)

Ang sakit o pagdurusa ng isang tao ay hindi lubos na iba sa akin, sapagkat sa pamamagitan ng aking katawan, nagiging posible na maintindihan at maranasan ko ang dinaranas ng ibang tao. Iba ang katawan ko sa katawan niya at iba rin ang pagtanggap namin sa sakit mula sa bawat isa. Ngunit dahil nilalang kami sa laman, nagagawa naming makipagugnayan sa bawat isa. Kasabay dito, ang pag-iintindi namin, na sa pamamagitan ng pag-uugnay namin sa bawat isa, sa pamamagitan ng aming laman, ay dumarating kami sa pagkakaiba at pag-uugnayan sa isa't isa.

\section{Etika ng Espasyo at Kalayaan}

Isang mahalagang etikal na ideya na ipinapahayag ni Merleau-Ponty sa kaniyang pilosopiya ng paglalaman ang kahalagahan ng pagbibigay ng espasyo at panahon para sa sarili at sa iba. Itong pagbibigay espasyo at panahon ay mapapansin sa kaniyang paglalahad ng kabalintunaan sa pagitan ng immanence at transcendence sa pagkakamalay.

Thus there is a paradox of immanence and transcendence in perception. Immanence, because the perceived object cannot be foreign to him who perceives; transcendence, because it always contains something more than what is actually given. ${ }^{12}$ (Mayroong kabalintunaan ng immanence at transcendence sa pagkakamalay. Immanence, dahil ang bagay na namamalayan natin ay hindi maaring maging ganap na ibang-iba sa kaniya na namamalayan ang mga bagay. Ang pagkakamalay din ay transcendence dahil lagi ito

11 Taylor Carman, Merleau-Ponty (New York: Routledge, 2008), 141.

12 Merleau-Ponty, $P P, 16$.

(c) 2017 Christian Joseph C. Jocson and Marvin Einstein S. Mejaro https://www.kritike.org/journal/issue 21/jocson\&mejaro december2017.pdf

ISSN 1908-7330 


\section{PILOSOPIYA NG LAMAN NI MERLEAU-PONTY}

nagbabahagi ng lubos-lubos na nilalaman, lumalagpas

kung ano ang namamalayan.)

Ang kamalayan para kay Merleau-Ponty ay naglalaro sa pagitan ng paglapit at paglayo. Ang namamalayan nating mga bagay ay hindi maaring maging malayong-malayo sa ating kamalayan, na darating na sa punto na walang pagkakataon na malaman at maabot natin. Ang mga bagay na namamalayan natin ay nakakasalubong natin na mukhang kilala, ngunit sa pagkakakita natin sa mga bagay ng ating kamalayan, nararanasan natin na may mga pagkakataon na nakakalaya ang mga bagay sa ating pagkakagapos sa kanila.

"Ang pag-aalam ay isang pamamaalam." Ang pagkakamalayan ay laging naglalaro sa magkabilang-dulo ng mga aspekto na kaya nating malaman at mga aspekto na lumalagpas sa ating kakayahan. Mahalaga rin na bigyan-pansin, na ang malimit na dapat na maging katapusan ng kamalayang pag-aalam ay ang ugali na hinahayaan ang bagay na mamalayan siya kung ano mismong bagay.

Binibigyan-diin ni Merleau-Ponty na ang pagrerespeto ng pagkakaiba ng bawat isa ay nagpapanatili ng pagiging sarili ng sarili at pagiging iba ng ibang tao. Anomang gawain na pinipilit ang sarili sa ibang tao ay nagdudulot ng karahasan hindi lamang sa katauhan ng ibang tao ngunit kasama rin ang sarili sa nakakaranas ng karahasan. Ang pagkakamalay ay masasabi natin na parang pagbabalanse sa pagitan ng pagkakasama at pagkakaiba, paglapit at pagkalayo, at immanence at transcendence.

Ang pagrerespeto sa pagkakaiba ng bawat isa ay nagiging haligi rin ng sarili ng bawat isa. Ngunit, hindi dapat humantong ang pagkakaiba sa pagkamanhid, na ang labis na layo natin sa bawat isa, wala na tayong pakialam sa kalagayan at katauhan ng bawat isa.

"Ang tinatawag ba natin na laman ay nasa loob nasa labas." Hindi lubusang nasa labas o ganap na nasa loob ang ipinahihiwatig ni MerleauPonty patungkol sa laman, sapagkat ang sinasabi niyang laman ay kapwa nagpapahiwatig ng labas at loob. Sa madaling salita, ang laman ay laging nakaturo sa sarili at sa ibang tao; ang laman ng aking laman ay nag-uugnay sa akin sa laman ng iba pang laman.

Ang Pranses na terminong 'milieu' ay mas malinaw na naipapahayag ang ideya ng Meron kay Merleau-Ponty. Ang pagtalunton sa etimolohiyang salitang milieu ay nagmula sa mi na gitna at lieu na lugar. Itinuturing ng mga iskolar kay Merleau-Ponty ang ideyang ito bilang kaniyang nosyon ng hyperdiyalektiko na humahagilap sa malawakang ideya ng diyalektikang pilosopiya. Mula sa nosyong ito ng hyperdiyalektika na natutukoy ang Meron sa isang purong estado, bagaman ang Meron, ay parating na sa 
sagandaan ng maraming meron. Kung gayon, bumubuo ang mga bahagi ng aking katawan ng isang sistema, kaya't ang aking katawan at ang iba pa ay isang kabuuan, kambal na bahagi nag-iisa at magkamukhang penomenon. ${ }^{13}$ Ngunit, maoobserbahan din na ang urong-sulong na pag-uugali ng isang lamang umiiral kay Merleau-Ponty ay nagpapamalas din ng isa pang pundasyon sa kaniyang ideya ng etikal na kalayaan.

\section{Kalayaan: Ang Pagbabahagi ng Espasyo at ng Panahon}

It seems to me that we can also say of other institutions that they have ceased to live when they show themselves incapable of carrying on a poetry of human relations that is, the call of each individual freedom to all the others. ${ }^{14}$ (Sa aking pananaw, masasabi na ang mga institusyon ay napatigil na sa kanilang mga gawain kapag hindi na nila kayang bigkasin ang tula ng pagkakaugnay ng bawat tao. Ito ang tinatawag natin na tawag ng kalayaan ng bawat isa sa bawat isa.)

Kailangang hayaan ang iba sa sarili natin, ipakita kung paano niya gusto ipahayag ang pagkakaiba niya nang hindi umiikot sa sariling larawan. Ang pag-iwas na hindi madaganan ng sarili ang katauhan ng ibang tao ay masasabi natin na nagpapahayag ng konsepto ng etikal na buhay at kalayaan para kay Merleau-Ponty.

Ang kamalayang pagtanaw para kay Merleau-Ponty ay hindi lang tungkol sa paglapit ngunit sa ating paglayo. Dito, mayroon tayong natatanaw na bago. Itong pananaw ni Merleau-Ponty ay nakatuon din para sa pagiintindi ng ating mga sarili, sapagkat ang pagkilala ng ating sarili at ng sarili ng iba pang tao ay hindi nakasalalay sa ating kakayahan lamang na lumapit. Bahagi ng ating kakayahan na matanaw at maintindihan ang mga tao at bagay sa ating paligid ay nakabase sa ating pagtanggap na hindi natin malalaman ng ganap ang mga bagay.

Dito ipinapakilala ni Merleau-Ponty ang konsepto ng chiasm bilang isang mahalagang aspekto ng ating pagtanaw at paglalaman sa mundo ng laman. Para kay Merleau-Ponty, ang chiasm ay ang solusyon niya sa mga kinagawiang mga magkatungaling mga konsepto at ideya sa pilosopiya. Mula sa paghihiwalay ng labas at loob, katawan at isip, at ang sarili at ang iba, sa pamamagitan ng konsepto ng chiasm ni Merleau-Ponty na ang kanilang paghihiwalay ay isang diyalektiko na pumupulupot sa bawat isa.

${ }^{13}$ Ibid., 412.

${ }^{14}$ Merleau-Ponty, $\operatorname{Pr} P$.

(c) 2017 Christian Joseph C. Jocson and Marvin Einstein S. Mejaro https://www.kritike.org/journal/issue 21/jocson\&mejaro december2017.pdf

ISSN 1908-7330 


\section{PILOSOPIYA NG LAMAN NI MERLEAU-PONTY}

Sa pamamagitan ng konsepto ng chiasm, maaari natin makita na ang relasyon ng sarili at ang Iba ay hindi ganap na paghihiwalay o pag-iisa, ngunit ang may ginagamit na salita ni Merleau-Ponty ay may magkabahaging pagiging. ${ }^{15}$

Ngunit kung titingnan ang nosyon ng espasyo, mapapansin na hindi lamang ito siyentipiko o epistemolohikal na ideya ng laman. Bagaman hindi niya tuwirang tinukoy ito sa kaniyang diskusyon tungkol sa ideya ng etikal na laman, mauunawaan na mahalagang konsiderasyon dito ay ang pagusapan ang nosyon ng laman at interkorporealiti. Ang hyperdiyalektika ni Merleau-Ponty kaiba sa diyalektika ni Hegel na humahagilap hindi para ikahon ang iisa at nagkakaisang layon ng Absolutong Geist. Ang hinahagilap na kaalaman ni Merleau-Ponty ay hindi lamang pagtatalaban ng mga abottanaw kundi ang paghahanap sa sala-salabin na mga abot-tanaw.

The solution to the problem of other bodies must be found within the identity within difference structure of reversibility. Here the Other functions as my mirror: he de-centers me, lets me see myself from another vantage. I do not coincide with the Other, but this experience of my being is not the undisclosable secret Sartre would make of it, either. ${ }^{16}$ (Ang solusyon sa problema ng pagiging ng ibang mga nilalang sa laman ay makikita sa pagkakaisa sa gitna ng pagkakaibang istruktura ng pagbabahagi. Dito ang Iba ay nagiging paraan kung paano ko nakikita ang aking sarili sa isang bagong pananaw-nilalayo niya ako mula sa aking sarili na parang tulad ng isang salamin. Ngunit, hindi dumarating sa punto na ang sarili at ang Iba ay nagiging isa, sa katulad rin na paraan, hindi naman masyadong makahiwalay ang sarili at ang Iba na walang na maaring posibilidad ng pakikitungo sa bawat isa, tulad ng pahiwatig ni Sartre.)

"Ang laman na nilalaman ang mga bagay sa mundo ay nakikita rin na may kinalaman siya sa ibang laman sa mundo." Ang kaalaman ng isang umiiral na laman ay parating patungo sa tawag na makiisa o ang pagkakaroon ng pakialam patungkol sa kalagayan ng iba pang umiiral na laman. Ang isang korporeal na tao ay isang tao na may pakialam.

\footnotetext{
${ }^{15}$ Cf. Maurice Merleau-Ponty, The Visible and the Invisible, trans. by Alphonso Lingis, ed. by Claude Lefort (Evanston, Illinois: Northwestern University Press, 1968), 214.

${ }^{16}$ M.C. Dillon, Merleau-Ponty's Ontology, 2nd ed. (Illinois: Northwestern University Press, 1988), 168.
}

(c) 2017 Christian Joseph C. Jocson and Marvin Einstein S. Mejaro https://www.kritike.org/journal/issue 21/jocson\&mejaro december2017.pdf ISSN 1908-7330

(c) BY-NC-ND 
Ang kalayaan sa pilosopiya ni Medeau-Ponty ay nagpapamalas ng isang uri ng pag-uugali na may respeto sa Meron at mga ibang meron sa paligid nito. Habang mas nababatid ko ang isang hindi-destruktibong diyalektika ng sarili at ng Iba, mas nagagawa kong mapalaya ang sarili sa mga kalasag ng mismong sarili. Ang daigdig ng laman at ang presensya ng iba pang mga umiiral sa aking paligid ay nag-iimbita sa mas malawakang ontolohikal na pagtanaw. Ang pagiging malaya ay ang pagtingin nang lagpas sa sarili bilang mas mataas sa sarili ngunit nasa relasyon ng isang mundo na nagpapalawig sa posibilidad ng Meron. Ang pagbukas ng sarili sa interkorporeal na daigdig ay ang tunay na kalayaan para kay Merleau-Ponty.

The Graduate School, University of Santo Tomas, Philippines

\section{References}

Carman, Taylor, Merleau-Ponty (New York: Routledge, 2008).

Cutrofello, Andrew, Continental Philosophy (New York; London: Routledge, 2005).

Dillon, M.C., Merleau-Pontv's Ontology, 2nd ed. (Illinois: Northwestern University Press, 1988).

Macke, Frank J., "Seeing Oneself in the Mirror: Critical Reflections on the Visual Experience of the Reflected Self," in Journal of Phenomenological Psychology 36:1 (2005).

Merleau-Ponty, Maurice, Phenomenology of Perception, trans. by Colin Smith (London; New York: Routledge, 1958).

The Primacy of Perception, ed. by James M. Edie (Illinois: Northwestern University Press, 1964).

The Visible and the Invisible, trans. by Alphonso Lingis, ed. by Claude Lefort Evanston (Illinois: Northwestern University Press, 1968).

Rae, Gavin, "Hegel, Alienation, and the Phenomenological Development of Consciousness," in International Journal of Philosophical Studies 20:1 (2012).

Singer, Peter, Hegel: A Very Short Introduction (Oxford: Oxford University Press, 2001).

Stern, Robert, "Is Hegel's Master-Slave Dialectic a Refutation of Solipsism?" in British Journal for the History of Philosophy 20:2 (2012). 